\title{
ANALYTIC HIERARCHY PROCESS AND CHOQUET INTEGRAL COMBINED WITHIN NON ADDITITIVE ROBUST ORDINAL REGRESSION FOR THE SELECTION OF SOCIAL HOUSING INITIATIVES
}

\begin{abstract}
Keywords: interactions, AHP, Choquet Integral.

\section{Introduction}

The study develops an integrated approach for resource allocation in Social Housing (SH) initiatives in Italy. The demand for $\mathrm{SH}$ has emerged all over Europe and it is exponentially increasing.
\end{abstract}

\section{Literature Review}

The Choquet integral is the most well-known and most used MCDA methodology taking into account interactions between criteria even if it shows two main problems: 1) the necessity to determine a capacity that assigns a weight not only to all single criteria but also to all subsets of criteria; 2) the necessity to express on the same scale evaluations on different criteria.

\section{Hypotheses/Objectives}

This research aims to test an innovative MCDA framework for an urban problem.

\section{Research Design/Methodology}

To handle the first problem (section 2) we adopt the recently introduced Non Additive Robust Ordinal Regression (Angilella et al., 2010) that takes into account all the capacities compatible with the indirect preference information provided by the DM. With respect to the second problem, we adopt a recent proposal to use the AHP to build the common scale on which the evaluations have to be expressed.

\section{Data/Model Analysis}

We involved in the process real DMs, who granted access to restricted data, thus enhancing the reliability of the results.

\section{Limitations}

The case study concerns the simulation of a process aimed to select interventions of adjustment for properties envisaging a housing supply for "weak" segments of the population in Turin. 


\section{Conclusions}

The proposed framework seems promising, in particular because of the presence of a certain number of interacting criteria and of a high number of alternatives.

\section{Key References}

Angilella, S., Greco, S., \& Matarazzo, B. (2010). Non-additive robust ordinal regression: A multiple criteria decision model based on the Choquet integral. European Journal of Operational Research, 201(1), 277-288. 